\title{
A case of Adams- Oliver Syndrome
}

\author{
V. Manikandasamy ${ }^{1}$, Arumugasamy $\mathbf{S}^{2}$ \\ ${ }^{1}$ Dr. V. Manikandasamy, Assistant Professor, ${ }^{2}$ Dr. Shanmuga Arumugasamy, Assistant Professor, both authors \\ are affiliated with Department of Pediatrics, Velammal Medical College and Research Institute, Velammal \\ Village, Anuppanadi, Near Chinthamani Toll Gate, Madurai, Tamil Nadu- 625009, India.
}

Address for Correspondence: Dr. V. Manikandasamy, Assistant Professor, Department of Pediatrics, Velammal Medical College and Research Institute, Velammal Village, Anuppanadi, Near Chinthamani Toll Gate, Madurai, Tamil Nadu 625009, E-mail: bharathi4samy@gmail.com

\begin{abstract}
A late preterm, 2.3kg, Male child was born to P2L2 mother by Normal Vaginal Delivery. Baby had a large scalp defect, acrania with hypoplastic and absent digits. Acrania portion of the skull was covered by a thick membrane. X-ray skullshowed absence of skull vault, X-ray of extremities showed hypoplastic and absent digits. Neurosonogram was normal. Echocardiography done showed moderate PDA with left to right shunt. CT brain was normal. Child was diagnosed as a case of Adams- Oliver syndrome. It is an autosomal dominant disorder comprises aplasia cutis congenita with terminal transverse limb defects.
\end{abstract}

Key words: Aplasia cutis congenita, Hypoplastic and absent digits

\section{Introduction}

Adams and Oliver described eight members of a family with this disorder in 1945. More than 100 affected individuals have been reported[1]. AdamsOliver syndrome is an autosomal dominant disorder with aplasia cutis congenita and terminal transverse limb defects[2]. We report a case of Adams Oliver syndrome with typical skin and limb defects without any major internal organ anomalies.

\section{Case Report}

A late preterm, 2.3kg, Male child was born to P2L2 mother by Normal Vaginal delivery to nonconsanguineously married parents. Baby cried immediately after birth. Apgar was 5 and 8 at 1 min and 5 min respectively. No adverse perinatal events occurred during birth. Elder sibling was alive and healthy, had no scalp or limb defects. Both the parents had no scalp or limb defects. Cry and activity was good. Baby's vitals were within normal limits. Head circumference is $33 \mathrm{~cm}$. Baby had a large scalp defect, acrania with hypoplastic and absent digits. Acrania portion of the skull was covered by a thick membrane.

Systemic examination findings were within normal limits. Baseline investigations were within normal limits. On radiological examination skull showed absence of skull vault, and extremities showed hypoplastic and absent digits. Neurosonogram showed absence of any intracranial anomalies. CT brain taken showed normal intracranial structures. Baby had moderate PDA with left to right shunt in Echocardiography.

\section{Discussion}

In 1945, Forrest Adams and C. Peter Oliver from Minneapolis first reported this condition. This condition is transmitted by Autosomal dominant mode of transmission, with presence of vertex cranial defects resembling aplasia cutis congenita and terminal limb malformations. More than 100 affected individuals have been reported[3].

Manuscript received: $7^{\text {th }}$ September 2017

Reviewed: $16^{\text {th }}$ September 2017

Author Corrected: $24^{\text {th }}$ September 2017

Accepted for Publication: $30^{\text {th }}$ September 2017 
Case Report

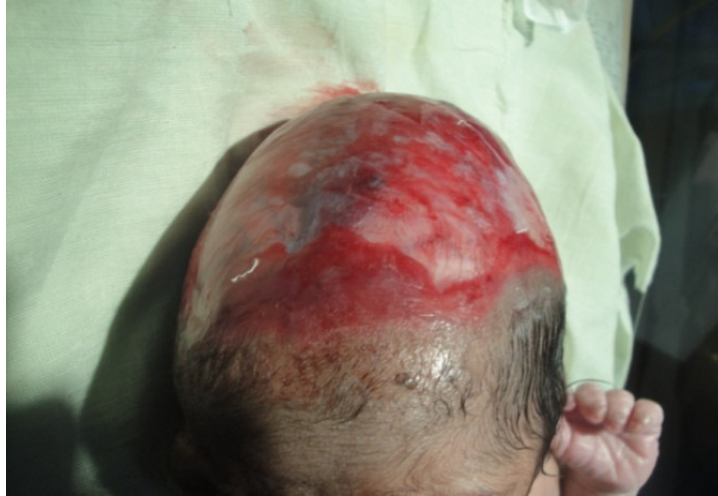

Figure-1: Acrania portion of scalp

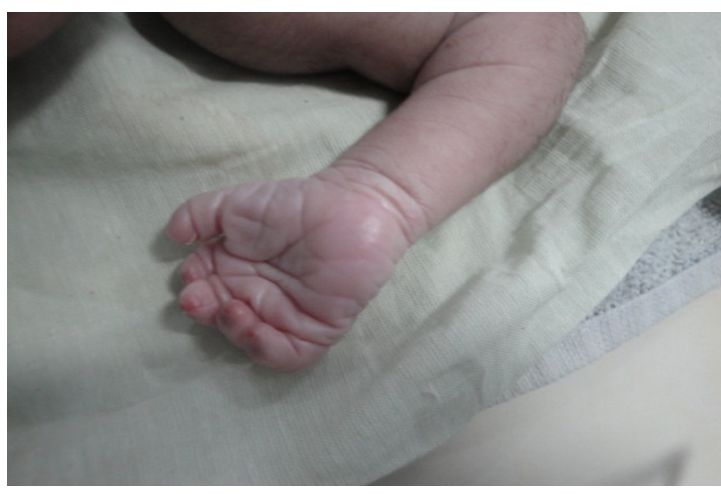

Figure-3: Hypoplastic digits in hand

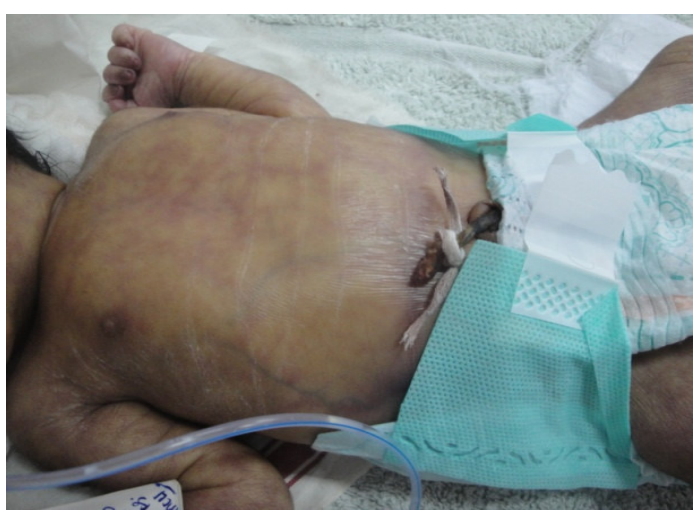

Figure-2: Cutis marmorata

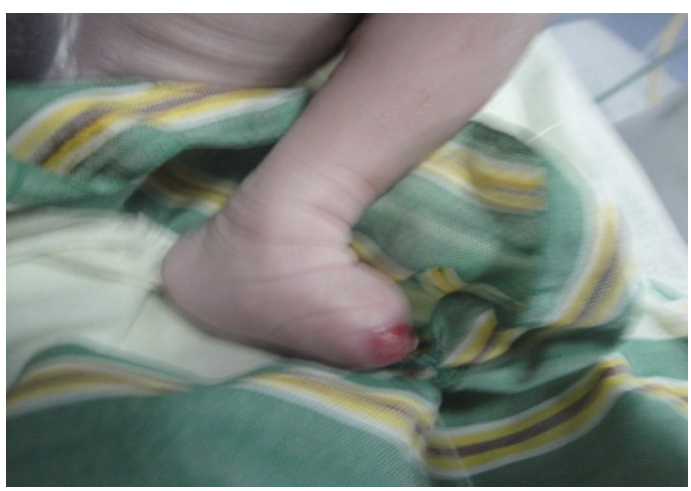

Figure-4: Hypoplastic digits in feet

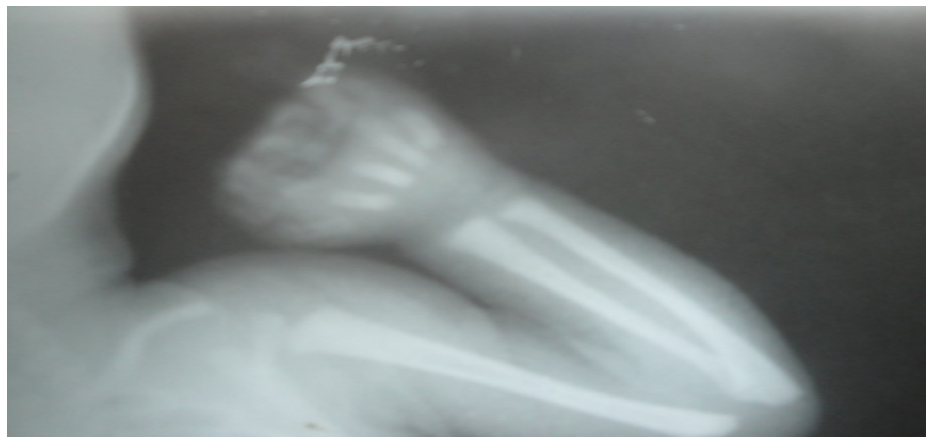

Figure-5: $X$ ray hand showing absent phalanges
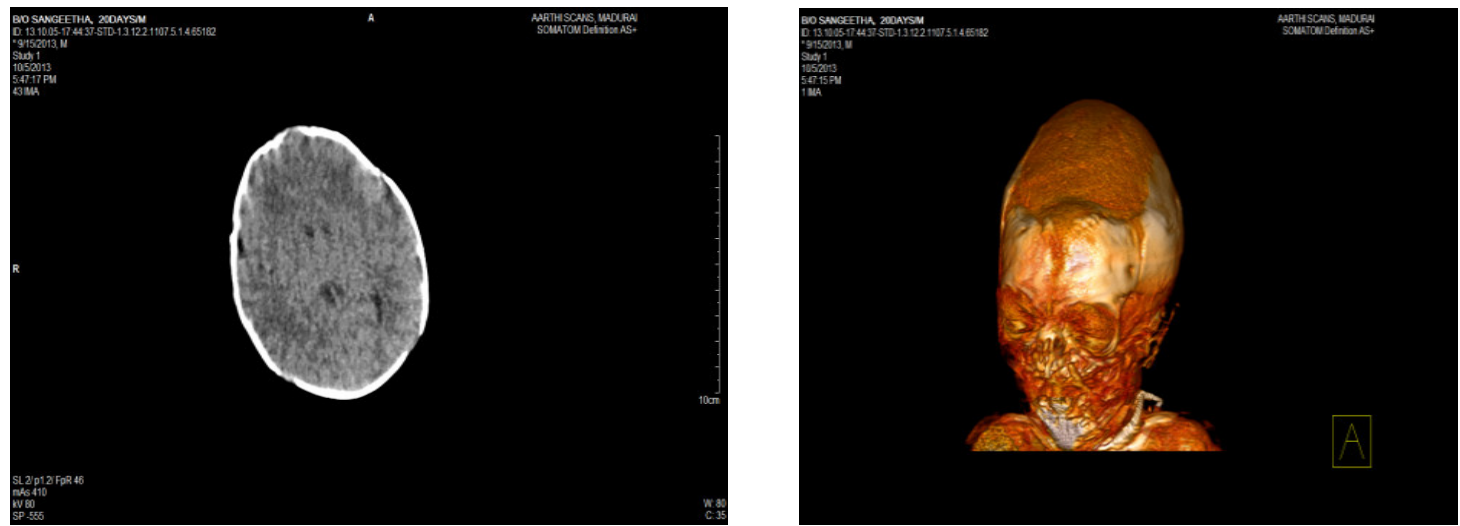

Figure- 6 and 7: CT brain showing normal intracranial structures 
Mild growth deficiency ( 3 rd to $10^{\text {th }}$ percentile) is seen. Aplasia cutis congenitais seen over posterior parietal region, with or without an underlying bony defect. These skin defects of the scalp can typically be found as solitary or multiple, round-oval hairless scars.

Terminal transverse limb defects, including those involvinglower legs, feet, hands, fingers, toes, or distal phalanges, short fingers and small toe nails. Cardiac defects are seen in $20 \%$ of affected individuals, including ASD, VSD, Coarctation of Aorta, obstructive lesions of the left heart, hypoplastic left and right ventricles, DORV, and DOLV. Cutis marmorata telangiectasia seen in $20 \%$ of cases.

An autosomal dominant inheritance pattern with marked variability in expression and lack of penetrance is seen in majority of cases. Gain-of-function mutations of ARHGAP31, a Cdc42/Racl GTPase regulator are responsible for the defects.

Autosomal recessive inheritance has been suggested in a few families. Recessive mutations in the dedicator of cytokinesis 6 (DOCK6) gene have been identified in some cases. Far more likely to have a severe phenotype with neurologic abnormalities and intellectual disability[1].

The pathophysiologic mechanism of these defects remains unclear, but several mechanisms have been proposed, including predisposition to amniotic rupture sequence, other forms of extrinsic trauma or compression, and vascular compromise. An intrinsic predisposition to interference with normal tissue development seems a likely etiology.

The association of cutis marmorata and the dilated and or tortuous scalp veins may be additional indicators pointing to an underlying predisposition to vascular compromise in "watershed areas such as the cranial vertex and limbs[2].

The male-to-female ratio of the affected cases is about 2:3 (in earlier reports of sporadic cases $=\mathrm{m}: \mathrm{f}=5: 13$; in earlier reports of familial cases $=16: 26$; in the present cases $=7: 3$; all together $=28: 42$ ).

Genetic counseling regarding the inheritance of this syndrome should be given to all parents. In genetic counseling autosomal dominant inheritance with great variability in expression of the syndrome of congenital scalp defect and distal reduction defects of the limbs should be stressed.

However, genetic counseling of families with a sporadic manifestation of the syndrome may be difficult. Anunaffected parent may represent a nonpenetrant individual. Thus, the unaffected parents of an affected child always have some risk of having another affected child. Ultrasonic examination might be indicated in all potential affected pregnancies.

Larger scalp defects with underlying defects of bone, where the superior sagittal sinus or dura are exposed there is an increased risk of hemorrhage or meningitis. Early surgical intervention with grafting is indicated. Cases in which the sagittal sinus or dura is not exposed, healing without need for grafting almost always occurs.

Several syndromes with congenital skinand limb defects have to be differentiated from the syndrome of scalp defect with perodactyly. Distal deficiencies occur in the aglossia-adactylia anomaly (Hanhart syndrome), Poland complex, and as a part of the limb defects in focal dermal hypoplasia.

A characteristic pattern of congenital scalp defects can be seen in the syndrome of scalp defects and postaxial polydactyly, the syndrome of scalp defects and split-hand defect, trisomy 13, and Johanson-Blizzard syndrome.

The skindefects of the amniotic band sequence are rarely found as localized defects on the scalp. In epidermolysisbullosadystrophica type Bart the defects are typically on the lower legs, and in focal dermal hypoplasia irregular atrophic areas are observed[4]. 


\section{Conclusion}

Adams Oliver syndrome is a rare disorder that can be associated with lethal major organ anomalies. Prognosis is good in majority of cases, especially those without any major organ anomalies. We report a case of AOS, most likely to be sporadic. The patient presented with isolated aplasia cutis congenital and terminal transverse limb defects.

Funding: Nil, Conflict of interest: None initiated, Perission from IRB: Yes

\section{References}

1. Jones KL, Jones MC, Campo MD. Smith's recognizable patterns of human malformations. Philadelphia: Elsivier Saunders; 2013.
2. Whitley CB, Gorlin RJ. Adams-Oliver syndrome revisited. Am J Med Genet. 1991 Sep1; 40 (3): $319-26$

3. Bamforth JS, Kaurah P, Byrne J, Ferreira P. Adams Oliver Syndrome: A family with variability in clinical expression. American Journal of Medical genetics 1994 Feb 15; 49(4): 393-96.

4. Kuster W, Lenz W, Kaariainen H, Majewski F. Congenital Scalp Defects With Distal Limb Anomalies (Adams-Oliver Syndrome): Report of Ten Cases and Review of the Literature. Americal Journal of Medical Genetics 1988; 31: 99-115.

\section{How to cite this article?}

V. Manikandasamy, Arumugasamy S. A case of Adams- Oliver Syndrome. J PediatrRes. 2017;4(09):580583.doi:10. 17511/ijpr.2017.i09.07. 